\title{
Introduction: Moral Enhancement
}

\author{
Andrea Lavazza ${ }^{1} \cdot$ Massimo Reichlin $^{2}$
}

Published online: 8 February 2019

๑) Springer Nature B.V. 2019

The repeated use of a faculty for a given purpose generally involves the improvement of this faculty. For our ancestors, a good example could be speed or resistance in running, as they had to hunt animals and protect themselves by escaping predators. Intellectual faculties also tend to improve with use, for example basic mathematical skills could gain speed and flexibility in those who had the task of managing an agricultural warehouse, where they had to add or remove different quantities of wheat. Over the course of history, it has been understood that targeted practice can enable greater and more specific improvements. Interestingly, the champions who competed in the ancient Olympics trained in very rudimentary ways compared to today's amateur athletes, while in philosophical academies intellectual exercise had already reached very high levels, which would still be considered excellent today. And this shows that different kinds of enhancement can move at a rather different pace.

The explicit ideal of the limited or continuous improvement of one's physical and cognitive endowments is not shared by all cultures. In any case, human beings have often tried to improve their character, dispositions, motives and behavior. In most societies, this process is what today we call socialization and moral education and was constituted by an often authoritarian or at least paternalistic kind of formation in which the family, the clan, the extended community or the state tried to make the individual internalize the rules of the group. For those who did not conform to those rules, there were forms of re-education, most often punitive. This did not prevent many people, although socialized in a specific culture, from individually choosing to improve themselves morally through meditation, the study of philosophy, religion and art, or by following the example of virtuous figures.

"Moral failures" were attributed to the wickedness of the subjects in question, the education system, the circumstances

Andrea Lavazza

lavazza67@gmail.com

Centro Universitario Internazionale, Arezzo, Italy

2 Vita-Salute San Raffaele University, Milan, Italy that overwhelmed the subjects' will, the subjects' personal limitations or social and psychological mechanisms at work in particular situations. Given such-actual or only potential-moral failures, the common choice was to remedy them by acting on one of the elements listed above. Generally, the idea of moral improvement was mainly linked to the restoration of a given average condition. An unfaithful husband could morally improve, just as society as whole, faced with the task of educating a new generation after a civil war filled with atrocities. This idea of improvement was connected to the view that, like other human faculties, the improvement of character, dispositions, motives and behavior occurred with a conscious and prolonged effort over time. In other words, there were no shortcuts to practice and commitment. The third idea underlying this conceptual framework was that the basic endowment of the human being was perfectly adequate for a satisfactory personal and social moral life. There was a need for effort and self-control, but everyone could be sufficiently "good" if they wanted.

This brief historical-conceptual excursus, though oversimplified, is helpful to frame and better understand the current debate on moral enhancement via biotechnologies. In fact, this form of enhancement-which we will shortly define with greater precision-is characterized by a reversal of the three claims that marked the traditional notion of moral improvement. Firstly, it is argued that the normal endowment of the human being on an emotional and cognitive level is not sufficient to develop character, moral dispositions, motives and behaviors adequate to the challenges of the time we live in. Secondly, and consequently, it is not so much a matter of remedying a "moral failure", but of raising one's moral abilities to a level never reached before, expanding them in unprecedented ways functional to our present needs. Thirdly, it follows that new tools are needed to forge higher-level moral choices and behaviors. And these tools are provided by contemporary biotechnologies, which promise to act directly on specifically localized brain functions, producing brain states that would translate into the moral behavior that we think is most appropriate. For this 
reason we can also speak of "moral neuroenhancement" as well as "moral bioenhancement".

Starting from these general premises, in the last decade a wide philosophical debate has begun on technological moral enhancement that can be easily divided into two strands of thought (Earp et al. 2018). The first strand of thought originates from the reflection of Ingmar Persson and Julian Savulescu, according to whom there is "an urgent imperative to enhance the moral character of humanity" and to develop research on the means of moral neuro-enhancement to this end (Ingmar Persson and Julian Savulescu 2008, 2012, 2013, 2015). The second strand of thought comes from Thomas Douglas (2008) and David DeGrazia (2014), for whom it should be morally permissible (Douglas) or morally desirable (DeGrazia) for individuals to willingly resort to certain forms of moral neuroenhancement, at least under certain circumstances.

As known, Persson and Savulescu (2012) have stated that humans are ethically unfit to face the challenges of the present age, which shows the need for moral enhancement. Their argument rests on the fact that today's humankind is facing two kind of threats "generated by the existence of modern scientific technology: the threats of weapons of mass destruction, especially in the hands of terrorist groups, and of climate change and environmental degradation" (Persson and Savulescu 2012, p. 1). According to the authors, humans are not morally equipped to face such global problems within a democratic system, especially when it comes to environmental problems. It is a fact that the scientific and political debate on global warming, with the related international conferences and formally signed protocols, trying to mobilize society from below with demonstrations and codes of conduct, have so far proved ineffective in limiting global gas emissions. The graph of these emissions, which has been dramatically rising since 1970 , shows only a small decrease in the years 2008-2009, coinciding with the economic crisis triggered by subprime mortgages. Nothing seems to curb our tendency to damage the environment in which we live, except the lower availability of income to spend.

For this and other reasons, Persson and Savulescu maintain that the improvement due to internalization and respect of moral doctrines "has been modest in comparison to the formidable improvement as regards scientific and technological knowledge". In short, there is a gap between what we actually do and what we could do morally, even though we are more ethically inclined than our ancestors: for example, we have expanded our moral circle encompassing many more people. In the light of the above, Persson and Savulescu envision the chance of moral enhancement by means of the latest biotechnological devices. In particular:

it is the motivational internalization of moral doctrines that we think could be sped up by means which the sci- entific exploration of the genetic and neurobiological bases of our behaviour might put into our hands. We call moral enhancement by such means moral bioenhancement; possible examples of moral bioenhancement would be drug treatment and genetic engineering (Persson and Savulescu 2012, p. 107).

They also clarify that the core moral dispositions are "altruism and a sense of justice as it primarily manifest itself in tit-for-tat." By defining them as moral dispositions, the authors assume that "by themselves, they always issue in a morally correct treatment of the individuals to whom they are directed" (Persson and Savulescu 2012, p. 108).

Hence a basic definition of neuroenhancement:

(Def1) Any change in a moral agent, A, effected or facilitated in some significant way by the application of a neurotechnology, that results, or is reasonably expected to result, in A's being a morally better agent (Earp et al. 2018, p. 168).

Other, more specific definitions, have been proposed, for example by Lavazza (2017):

(Def2) moral bioenhancement is an intervention on the nervous system or the precursor genes of the nervous system aimed at making the individual's phenotype and/or behavioral profile more altruistic (meaning altruism as a willingness to consider the interests of others as equal to one's own) and more oriented to justice (understood as the equal treatment of similar cases), compared to before the treatment. This means that decreasing antimoral emotions/dispositions is also part of moral bioenhancement.

The first objection to the idea of enhancement has come from fears related to changing human nature and violating the natural order. In addition, there is the skepticism about the practical possibility of acting as conceptually formalized by philosophers. However so far, there is no "pill of morality", but there are various strands of research striving to contribute to moral enhancement. They range from the administration of hormones like oxytocin, which can increase prosocial attitudes, to chemical action on the levels of serotonin and testosterone in order to decrease aggression and the propensity to harm others. There are non-invasive or minimally invasive electromodulation techniques to affect brain activity in order to increase self-control or improve moral judgment. Other psychoactive substances such as methylphenidate or lithium seem capable of acting as inhibitors of violent or sexually inappropriate behavior.

But even in the face of these substances and these techniques, which are the result of research oriented primarily to other clinical purposes, it seems necessary to identify precisely what brain/mental functions underlie 
moral dispositions and moral behavior, so that there may be a truly targeted and effective intervention. Secondly, it should be specified in what direction the upgraded functions should be implemented. In other words, it should be clarified what "moral betterness" means. The case of empathy, widely discussed in recent years, has become paradigmatic. The prosocial disposition that derives from identifying with others seems to be rooted in ancient innate brain mechanisms that tend to make us privilege sensitivity and action in favor of those who are close to us and whose discomfort or suffering we can experience directly. If we enhance this "function", the circle of our moral concern risks remaining restricted to small groups with whom we have face to face interactions, to the detriment of a more rational and enlarged consideration of the needs and priorities of others on a larger scale. Typically, the global problems mentioned above could paradoxically find less attention in the empathically empowered.

For this reason, there is consensus on the fact that moral neuroenhancement should ideally involve the improvement of higher-order abilities, with a flexible, reasonable, and appropriate response to the various (or even unpredictable) circumstances and needs that may arise. For example, we may agree that it would be good to decrease aggression, generally speaking, but a morally wholehearted individual also ought to be able to react firmly to defend vulnerable subjects and not be overly submissive when it comes to collectively deciding courses of action that could be morally negative. In this sense, the appreciation and strengthening of specific functions and faculties can be controversial. If indeed the general idea of moral bioenhancement is not linked to a specific conception of the good, but to an ability to act morally that implies moral disagreement, whether and to what extent it one should act on certain functions also seems to inevitably refer to substantive conceptions of the right and the good, not only in a procedural sense.

Another topic subject to discussion and criticism in relation to moral enhancement via biotechnology concerns features that are properly philosophical. In fact, one may ask whether moral bioenhancement is morally justified, permissible and desirable - or whether it should even be compulsory. Here, on the one hand, there are paternalistic and liberal positions (to different degrees), which privilege the protection of society or the autonomy of the individual. On the other, there are positions claiming that all interventions of moral enhancement ultimately "undermine our freedom by reducing our options to act immorally", as they fail to address the intellectual aspects of moral decisions (Verkiel 2017; cf. Harris 2011, 2016; Sparrow 2014). The criticism that supports the restriction of freedom emphasizes the loss of autonomy produced by moral bioenhancement, regardless of whether it is imposed or voluntarily chosen. Indeed, effective moral enhancement would prevent us from performing morally undesirable actions and having morally undesirable motives.

This important criticism is based on the idea that a direct moral neuroenhancement of functions or capacities (both in a positive and in a negative sense, as a limitation of a given function or capacity) would limit our agency, as in that case we would not act for moral motives and reasons we give our rational assent to, but only for the effect of a substance or technique modulating that function or capacity. The issue of the loss of freedom has been answered by emphasizing the result obtained, which would overcome and justify the limitation to our autonomy. Just as we would agree to limit the physical freedom of a terrorist who is about to throw a bomb in a concert hall in order to save the spectators' lives, so we could accept the partial loss of moral autonomy in exchange for moral neuroenhancement. Also, moral bioenhancement seems to benefit everyone while harming no one, unlike other forms of physical or cognitive enhancement.

The objection related to moral agency - which also mentions a Kantian objection against bioenhancement - is more thorny and is the reason why scholars tend to prefer an indirect form of moral bioenhancement, also called secondorder. On the one hand, the direct pharmacological empowerment of a faculty, as mentioned earlier in the case of empathy, can provoke a behavior that is positive in its effects but not moral in its motivations, because the individual does not really choose to be more empathetic (although a conscious choice to be enhanced could be a moral reason for the subsequent behavior). On the other hand, it can expose itself to the criticism of misfiring, that is, of not being functional to a real moral improvement (because, for example, too much empathy makes us short-sighted to the needs of those who are far away).

If instead the objective is to support learning and moral progress pursued with traditional methods, such as reading novels and works of philosophy, then bioenhancement will not bypass the moral reasons for which an individual acts. Rather, it will indirectly strengthen them, empowering faculties that are part of the process of moral choice and action. This is the moral flexibility mentioned above, which is the preferential objective of moral improvement (also via biotechnology). Moreover, faced with the inevitable (indeed, perhaps desirable for the progress of substantive conceptions) moral disagreement on what to strengthen in moral enhancement, one might privilege a form of "procedural moral enhancement" that aims to improve decision-making rather than specific views, motivations or moral provisions. In other words, one could aim (despite operational difficulties) to strengthen perspectivetaking understood as a moral epistemic capacity (for example the search for impartiality), instead of acting on affective aspects or particular facets of one's moral capacity. In particular, it has been suggested that one could act on the 
diminution of the sense of self (for example through psychoactive substances), which would reduce self-interested motivations.

This brief overview of the main themes addressed in the moral bioenhancement debate (cf. Buchanan 2011; Wiseman 2016) shows the meaning and usefulness of this collection of timely and well-argued essays. This special issue of Topoi presents a series of contributions that push the discussion forward, both refining and extending the proposal for a moral enhancement via biotechnology, and considering some objections raised to moral neuroenhancement, also formulating new criticisms of the general idea and of its possible forms of implementation.

Ingmar Persson and Julian Savulescu, in their contribution "The Duty to be Morally Enhanced", argue that we have a duty to develop and apply safe and cost-effective means to increase the probability that we shall do what we morally ought to do, including biomedical means of strengthening the central moral drives of altruism and a sense of justice. According to the authors, this is necessary because commonsense morality has a evolutionary origin and it has to be revised to serve the needs of the contemporary globalized society.

Andrea Lavazza, in his article "Moral Bioenhancement Through Memory-editing: A Risk for Identity and Authenticity?", focuses on a new possible means of neuroenhancement, targeting people with lower propensity to trust and altruism. Lavazza's idea is that, based on attachment theory, people with insecure attachment are less inclined to prosocial behavior. We know that these people are influenced by negative childhood memories and this negative emotional component may be reduced by the administration of propranolol, thereby improving prosocial skills. But this intervention raises questions about the preservation of identity and personal authenticity, which the author discusses.

Antonio Diéguez and Carissa Véliz, in their paper "Would Moral Enhancement Limit Freedom?", discuss the objection that moral enhancement would inhibit our "freedom to fall". In this view, the possibility of morally bad actions is seen as an important part of our freedom, which would therefore be compromised. The authors' response is that a well-educated person, without bioenhancement, will hardly have "moral falls", but this does not make them any less free. Secondly, enhancement would broaden the range of possibilities for moral action, compensating for the loss of the "freedom to fall".

Michelle Ciurria, in her contribution "Is There a Duty to Use Moral Neurointerventions?", refutes the criticism of moral bioenhancement as a threat to the self, which allegedly does not participate actively in the process. Ciurria argues that some neurointerventions are low risk, do not threaten the self and can even be considered a duty, provided they comply with three conditions: (1) pass pre-clinical and clinical trials; (2) do well in post-clinical studies; (3) be subject to regulations protecting informed consent.

Anton Vedder, in his paper "An Obligation to Enhance?", reviews some formal features of possible obligations to be enhanced. The starting point is that there may be obligations to be enhanced in the absence of good moral reasons and therefore such obligations are not morally desirable or right. Another point that the author explores concerns the person who is subjected to the obligation or the duty of enhancement: it is the person on whom the enhancing treatment is performed, or the controller/ operator of the enhancement?

Massimo Reichlin, in his article "The Moral Agency Argument Against Moral Bioenhancement", underlines the radical difference between traditional and biotechnological ways of producing moral progress. He claims there is a risk that moral bioenhancement may cause serious negative effects on our moral agency, by producing a substantial loss of freedom and capacity of authentic moral behavior, by affecting our moral identity and by imposing a standard conception of moral personality.

Fabrice Jotterand and Susan B. Levin, in their contribution "Moral Deficits, Moral Motivation and the Feasibility of Moral Bioenhancement", criticize Persson and Savulescu's position, which, for them, pathologizes human behavior in relation to weak moral motivation. Jotterand and Levin claim that bioenhancement does not increase moral motivation because it only focuses on one component of it - namely, the affective dimension Based on Aristotle's virtue ethics, the authors offer a different account of moral psychology and moral development.

Jonathan Pugh, in his article "Moral Bioenhancement, Freedom, Value and the Parity Principle", defends non-cognitive moral bioenhancement against the criticism by which it allegedly hinders our "freedom of fall". The author considers an Aristotelian reading of the former and notes how this objection faces the problem of explaining why the value of moral conformity is insufficient to outweigh the value of the freedom to fall. Finally, Pugh considers the role of the "parity principle", which states that in assessing the moral permissibility of a neurointervention, we should ignore the nature of the intervention, and focus instead on the costs and benefits of the procedure, broadly construed.

Adriano Pessina, in his contribution "A Clay Person. The Promises of Moral Bioenhancement", highlights the risk that the human being might be treated as "something" to be perfected to the detriment of being "someone" to respect and not to manipulate. In addition, the author claims that values associated with the plurality of moral experience might be lost if moral personal growth is only sought through chemicals and new technology, thereby creating a new form of impersonal dependency.

Norberto Paulo and Jan Christopher Bublitz, in their paper "How (not) to Argue for Moral Enhancement: 
Reflections on a Decade of Debate", strongly criticize the project of moral bioenhancement because, in their view, the current proposals are non-persuasive as they fail to diagnose the often complex causes of contemporary moral maladies and are premised on methodological individualism. Paulo and Bublitz maintain that focusing on brain and mind moral bioenhancement neglects social and environmental factors. They therefore advocate a different way to tackle our pressing problems.

Karolina Kudlek, in her contribution "The Role of Emotion Modulation in Moral Bioenhancement Debate", develops a criticism of enhancement of moral motivation via direct emotional modulation. According to the author, neuroscientific and evolutionary-psychological perspectives seem to indicate that emotions don't fulfill the necessary requirements to serve as the vehicles of moral enhancement and we should, therefore, take into account the role and function of all the cognitive modules associated with moral decision-making.

Keisha Ray and Lori Gallegos de Castillo, in their article "Moral Bioenhancement, Social Biases, and the Regulation of Empathy", argue that biomedically inducing more empathy may diminish moral agency. The authors argue that this type of increase in empathy would not be effective for addressing empathy's vulnerability to certain biases that can undermine the important capacity to regulate empathy. The project of moral bioenhancement should therefore consider this challenge.

Mirko Garasic, in his contribution "Enhancement 2.0: Self-Creation Might not be as Lovely as Some Think", concentrates on Emotional Enhancers as manipulative tools and on the potential results of their implementation. The author argues that these types of emotional enhancers can produce risks involving sexual minorities. In light of this concern, he advocates a much more cautious assessment of those drugs and a broader assessment of what their social social acceptance would mean.

Bob Fischer, in his paper "Moral Bioenhancement Probably Won't Improve Things for Animals (and May Make Them Worse)", tackles the topic of moral enhancement in relation to how we treat animals. His position is that moral progress via biotechnologies could worsen the condition of animals for two reasons. First, there are cognitive mechanisms that lead us to give less consideration to animal interests than to human interests-not overridden by increased altruism; second, there are deep tensions between the interests of humans and animals that moral bioenhancement may well exacerbate.

\section{References}

Buchanan A (2011) Beyond humanity?: the ethics of biomedical enhancement. Oxford University Press, New York

DeGrazia D (2014) Moral enhancement, freedom, and what we (should) value in moral behaviour. J Med Ethics 40(6):361-368

Douglas T (2008) Moral enhancement. J Appl Philos 25(3):228-245

Earp BD, Douglas T, Savulescu J (2018) Moral neuroenhancement. In: Johnson LSM, Rommelfanger KS (eds) The Routledge handbook of neuroethics. Routledge, New York, p 166-184

Harris J (2011) Moral enhancement and freedom. Bioethics 25(2):102-111

Harris J (2016) How to be good: the possibility of moral enhancement. Oxford University Press, Oxford

Lavazza A (2017) Moral bioenhancement through memory-editing: a risk for identity and authenticity? Topoi. https://doi.org/10.1007/ s11245-017-9465-9

Persson I, Savulescu J (2008) The perils of cognitive enhancement and the urgent imperative to enhance the moral character of humanity. J Appl Philos 25(3):162-177

Persson I, Savulescu J (2012) Unfit for the future: the need for moral enhancement. Oxford University Press, Oxford

Persson I, Savulescu J (2013) Getting moral enhancement right: the desirability of moral bioenhancement. Bioethics 27(3):124-131

Persson I, Savulescu J (2015) The art of misunderstanding moral bioenhancement: two cases. Camb Q Healthc Ethics 24(1):48-57

Sparrow R (2014) Better living through chemistry? A reply to Savulescu and Persson on 'moral enhancement'. J Appl Philos 31(1):23-32

Verkiel SE (2017) Amoral enhancement. J Med Ethics 43(1):52-55

Wiseman H (2016) The myth of the moral brain: The limits of moral enhancement. The MIT Press, Cambridge (MA)

Publisher's Note Springer Nature remains neutral with regard to jurisdictional claims in published maps and institutional affiliations. 